\title{
Automatic Registration of 3D Camera Recording to Model for Leads Localization
}

\author{
Samir Alioui ${ }^{1}$, Martim Kastelein ${ }^{1}$, Eelco M van Dam ${ }^{1}$, Peter M van Dam ${ }^{1}$ \\ ${ }^{1}$ Peacs, Arnhem, the Netherlands
}

\begin{abstract}
The purpose of this research is to localize ECG electrodes from a $3 D$ photo and to reduce electrode misplacement related errors in the ECG interpretation.

A Kinect camera is used to take a $3 D$ photo, which is registered automatically to a model derived from $M R I / C T(M C)$. The registration algorithms use a sternum marker attached to the skin to enable an accurate and fast matching of the $3 D$ photo and the MC model. The algorithms have been compared to optimal manual registration. For all cases the automatic registration algorithms performed much better than the manual registration. The automatic registration of a $3 D$ photo with patient specific electrode positions will offer a novel way of preventing and correcting errors due to ECG lead misplacement.
\end{abstract}

\section{Introduction}

Accurate localization of the ECG electrodes on the surface of a patient is very important for inverse ECG ( $i$ ECG) methods, especially when only the 12 lead ECG is used [1]. To localize the electrodes accurately on the skin, markers can be used while recording a magnetic resonance imaging (MRI) or computed tomography (CT) [2], but this requires a costly MRI or CT scan. Consequently, multiple ECG recordings require multiple MRI/CT scans. A 3D photo, registered to the MRI or CT data or readily available model can support the localization of the ECG electrodes in a simple and cost effective way [3].

A 3D camera, like for instance the Kinect camera, offers such a fast and easy applicable method to determine the electrode positions on the chest. Over the past years specialized Kinect software has been developed and tested to accurately determine the electrodes. This software was successfully used in our recent research to localize the origins of ventricular arrhythmias [4]. However, the manual registration of the 3D Kinect photo to the 3D torso surface as derived from MRI/CT has been cumbersome. Automatic registration of the $3 \mathrm{D}$ photo is therefore important, because it will reduce effort and variability to localize the electrodes from the 3D photo by the operator.

The current study describes the method to automatically register the 3D torso photo to the torso model derived from MRI/CT (MC model). The validity of the result is estimated by calculating the distance differences between 3D photo and MC model.

\section{Methods}

The ECG electrode position localization involves 4 steps:

1) Recording of a 3D-photo

2) Analysis of the 3D-photo

3) Registration of the 3D photo to an torso model

4) Extraction of the ECG electrode positions

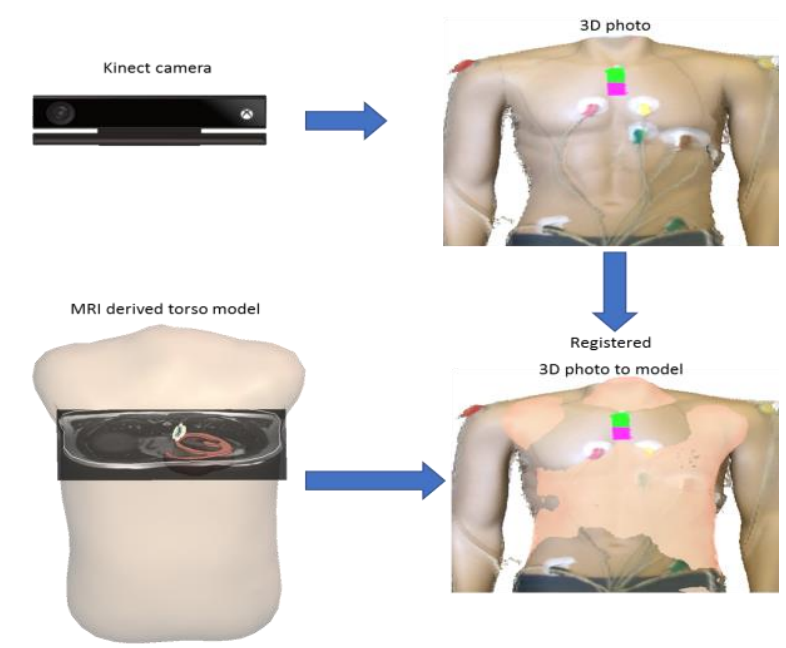

Figure 1 System to register a 3D photo to a model of the thorax. The CM model of the thorax is derived from MRI or CT. The visible electrodes enable an accurate localization of the electrodes after the registration of the 3 D photo.

\section{D-photo recording}

The Kinect camera is used to take a 3D-photo of the patient. The camera captures both color information and depth information and combines them into the 3D 'pixel' points representing the $3 \mathrm{D}$ photo [5].

The 3D-photo is passed through a filter and is pre-analyzed to enable the registration of the photo to a $\mathrm{CM}$ model of the torso. To grant the automatization and optimization of the recording and improve the registration process, a visual marker was introduced which supports the algorithms to analyze the relevant areas of the torso. This marker is placed at the top of the sternum (figure $2 \mathrm{~b}$ ). The $3 \mathrm{D}$ photo recording is automatically started after this marker has been detected by the software. 


\section{Analysis of the 3D-Photo}

The pre-analysis segments the torso based on the visual properties of the marker. The marker colors have been chosen such that its detection is easy on the skin. Moreover color and shape were chosen such that an initial estimated on the head-feet direction can be derived from the marker (figure 2a). The center of the rectangular marker is determined by computing the intersection of the diagonals. The vertical orientation, top/bottom, by using the colors from the recorded 3D photo. The left and right are detected using the normal on the marker plane (figure 2c) in combination with the known marker height and width (figure 2a).

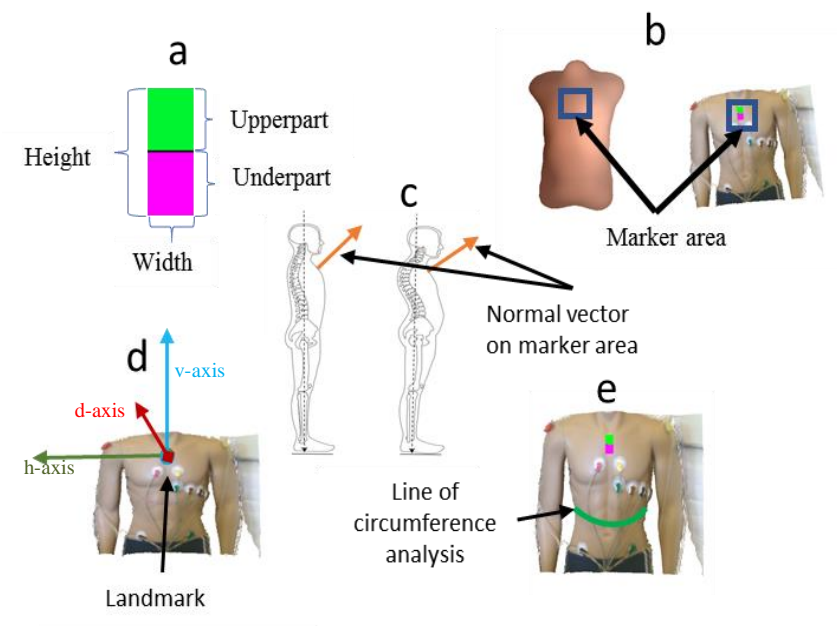

Figure 2: Different elements analyzed in the $3 D$ photo. a) the marker, $b$ ) the area where the marker needs to be placed, c) examples of the normal vector in the marker area, $d$ ) the coordinate system at the land mark (center of the marker), and e) determining the vertical torso direction using the circumference analysis.

Attaching the marker at the top of the sternum (manubrium) permits the detection of the sternum point representing the center of the marker on the $3 \mathrm{D}$ photo. The same point is also well defined in the CM model and serves as center of the coordinate system, the landmark, (figure 2d) for all next registration steps.

The marker is used to estimate the orientation of the torso by estimating the coordinate system at the landmark. To determine the vertical (v-axis) and horizontal (h-axis) axis of the coordinate system, the marker is virtually divided to four areas. The normal vector, which represents the curvature of the marker area (figure 2c), is the d-axis.

Using this coordinate system (figure 2d) the classification of the $3 \mathrm{D}$ photo pixels is processed into either a) chest element or b) no chest element. To determine the zone of interest for registration purposes the algorithm selects an area around the sternum. The points with limited depth variation on all circumferential lines (green line in figure 2e) from the landmark towards the feet are added to the area of interest.

A similar zone of interest is to be defined on the CM model to enable the registration of the photo to the model.

\section{Registration of the $3 D$ photo to the torso model}

The registration of the $3 \mathrm{D}$ photo to the model contains two main phases, the initialization and the actual registration.

Initialization The patient specific MC model also contains the top of the sternum location. This sternum location, the model landmark position, is used to calculate the model normal (figure 3a) indicating the curvature of the equivalent Peacs marker area (figure $2 \mathrm{c}$ ).

A second position downward from the landmark position on the model is required (figure $3 \mathrm{a}$ ). That point is called the balance point which is needed by the algorithms to converge into the best solution. The curve characterizing the torso surface grants the uniqueness of that point.

Registration: The registration process results from the interpolation of the transformation function that relates the MC Model to the 3D photo which is expressed as follows:

$$
\begin{aligned}
& \forall p(x, y, z) \in E_{1} \wedge \forall k(x, y, z) \in E_{2} \\
& \mathrm{k}_{\mathrm{i}}=\mathrm{T} \mathrm{p}_{\mathrm{i}},
\end{aligned}
$$

with $E_{1}$ the set of $3 \mathrm{D}$ photo $3 \mathrm{D}$ points, $\mathrm{E}_{2}$ the set of $\mathrm{MC}$ model 3D points and $i$ the index of the point in $\mathrm{E}_{1}$ or $\mathrm{E}_{2}$, and $\mathrm{T}$ is Transformation Matrix

The interpolation function is based on three parameters:

- Distance between the marker on 3D photo and its equivalent emplacement on the MC model. The point coordinates of $3 \mathrm{D}$ photo are expressed in camera space coordinates which is different from the space coordinates of the MC model. Therefor the 3D photo landmark is translated to match the landmark of the MC model.

- The angles between the normals in the two models. Initially the normals representing the curves of the marker planes are differently oriented in spite of representing the same area curve (figure $3 \mathrm{c}$ ).

- The definition of the balance point that converges to the best fitting: The left-right symmetry characterizes the marker area. The presence of external objects (electrodes for instance) on the chest and differences in MRI/CT and $3 \mathrm{D}$ photo recording environment influences the symmetry. The balance point is a control point that ensures the convergence to the best solution by avoiding the possible wrong solutions when only the normals would be used (figure 3d), as it permits the use of the curve characteristic of the torso surface.

After both landmark points have been matched by a translation of the $3 \mathrm{D}$ photo to the $\mathrm{MC}$ model, the registration continues by calculating the angle between the normal vectors from $3 \mathrm{D}$ photo and the MC model. This angle is used to rotate the 3D photo to align both normals. 


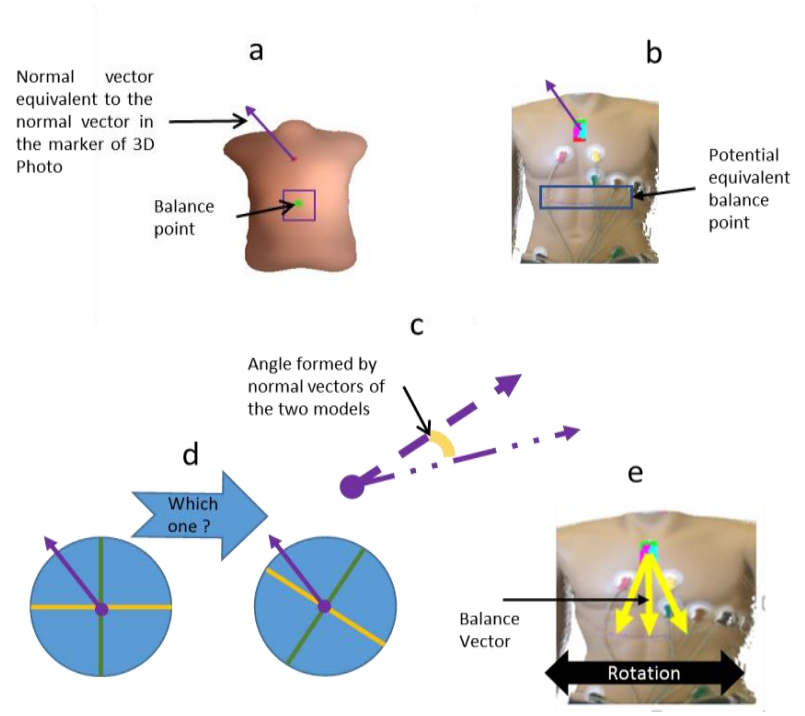

Figure 3: Different instances of initialization and torso characteristics used; a) normal at marker area, b) potential balance point, c) difference between $3 D$ photo and model normal, d) symmetry problem using only the normal, and e) potential optimal balance vector

The algorithm now uses the balance point (figure $3 \mathrm{e}$ ) to maximize the fitting. The fitting is optimized by iterating over all potential equivalent balance points in the $3 \mathrm{D}$ photo point (figure $3 \mathrm{~b}$ ). For each equivalent balance point the percentage of fitting is estimated by calculating the distance of all points in the area of interest on the $3 \mathrm{D}$ photo to the CM model. This phase will generate a space of solutions represented in the following graph.

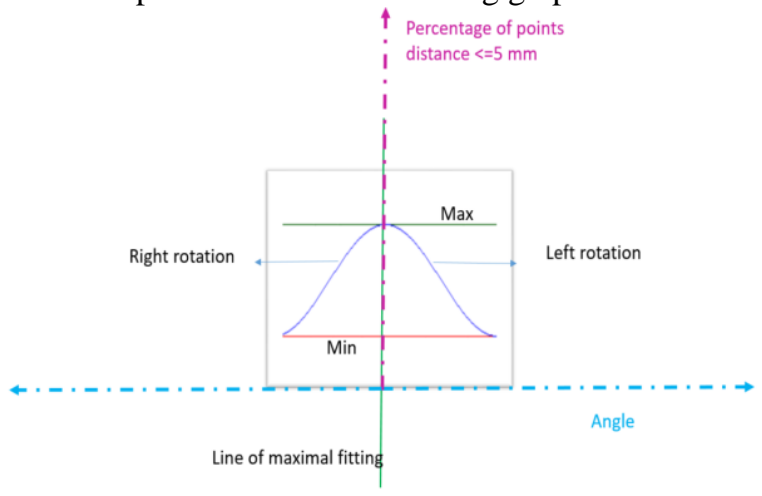

Figure 4: Graph of variation of percentage of fitting around the balance point.

The transformation delivering the maximal fitting is chosen (figure 5) by comparing the percentage of points contained by the area of interest in a range of $[0 \mathrm{~mm}$ $15 \mathrm{~mm}$ ] categorized in steps of $2.5 \mathrm{~mm}$ defining a system of contribution weight as represented in table 1 (table contribution weight). An acceptable improvement is the one with a score $>=50 \%$.
Table 1 The different categories for the distance between $3 D$ photo and the MC model. The weight for each category is listed in the right column. The total number of points (Ctot) is 21 .

\begin{tabular}{|c|c|}
\hline $\begin{array}{c}\text { Categories } \\
\text { [mm] }\end{array}$ & $\begin{array}{c}\text { category weight } \\
\text { [points] }\end{array}$ \\
\hline$\leq 2.5$ & 6 \\
\hline$\leq 5$ & 5 \\
$\leq 7.5$ & 4 \\
\hline$\leq 10$ & 3 \\
\hline$\leq 12.5$ & 2 \\
\hline$\leq 15$ & 1 \\
\hline
\end{tabular}

\section{Electrode Position localization}

The ECG electrode positions are manually localized in the 3D photo by visual identification. The user selects the ECG electrode by clicking on the area where it is located in $3 \mathrm{D}$ photo. The algorithm estimates the geographical center of the points representing the electrode. Finally that center will be projected onto the MC model (Figure 5).

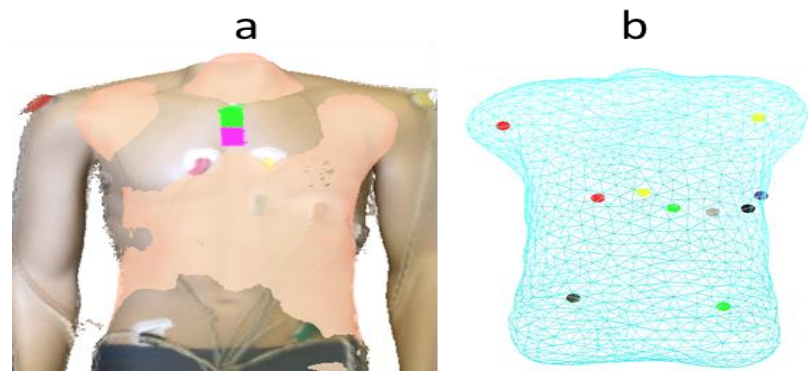

Figure 5: Final result of the registration (a) and the localization of the ECG electrodes (b). The electrodes are shown as colored dost on the triangulated torso mesh.

\section{Results}

The result are obtained from the comparison of 3D photos from different subjects referenced with index Patient number and of 3D photo from the same subject characterized by different ECG electrode positions. Those $3 \mathrm{D}$ photos are indexed with letters: $\mathrm{L}$ for lower, $\mathrm{H}$ for higher and $\mathrm{N}$ for normal positioning of the ECG electrodes.

Table 2 shows the difference between the manual and the automatic registration in terms of the fitting percentage differences within range of $\left[\begin{array}{llll}0 & \mathrm{~mm} & -15 & \mathrm{~mm}\end{array}\right]$ and categorizing steps of $2.5 \mathrm{~mm}$, also it gives the improvement gain from it.

The improvement score, $I S$, is calculated as follow:

$$
I S=\frac{1}{C_{\text {tot }}} \sum_{i=1}^{n}\left(\left(A_{i}-A_{i-1}\right)-\left(M-M_{i-1}\right)\right) C W_{i}
$$

where Ctot is the total number of weight points (see table 2 ), $\mathrm{A}$ is the automatic fitting result for category $i$, and $\mathrm{M}$ 
the manual fitting results. The category $i=0$ the fitting is by definition $0 . C W_{i}$ is the weight of category $i$ (see table 2).

Table 2 The comparison between manual and automatic registration of the CM model with the $3 D$ photo for the different distance categories.

\begin{tabular}{|c|c|c|c|c|c|c|c|}
\hline \multirow{2}{*}{$\begin{array}{l}\text { patient } \\
\text { ID }\end{array}$} & \multicolumn{6}{|c|}{$\begin{array}{c}\% \text { of the area less than distance } \\
\text { category in } \mathbf{~ m m}\end{array}$} & \multirow{2}{*}{ 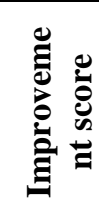 } \\
\hline & $\stackrel{n}{v i}$ & VI & $\frac{n}{\mathrm{~V}}$ & $\stackrel{\circ}{\mathrm{V} I}$ & $\frac{\dot{v}}{\mathrm{~V}} \mathrm{n}$ & $\frac{n}{\mathrm{~V} I}$ & \\
\hline M1 & 20 & 29 & 38 & 45 & 51 & 57 & \multirow{2}{*}{+5.2} \\
\hline A1 & 32 & 47 & 57 & 67 & 70 & 76 & \\
\hline $\mathrm{M} 2 \mathrm{~N}$ & 15 & 22 & 27 & 33 & 38 & 43 & \multirow{2}{*}{+6.5} \\
\hline $\mathrm{A} 2 \mathrm{~N}$ & 25 & 38 & 50 & 60 & 68 & 75 & \\
\hline M2L & 3.9 & 7 & 10 & 14 & 18 & 21 & \multirow{2}{*}{+12.4} \\
\hline A2L & 25 & 37 & 49 & 49 & 62 & 75 & \\
\hline $\mathrm{M} 2 \mathrm{H}$ & 6.3 & 10 & 14 & 17 & 20 & 23 & \multirow{2}{*}{+13.12} \\
\hline $\mathrm{A} 2 \mathrm{H}$ & 30 & 45 & 57 & 69 & 79 & 86 & \\
\hline
\end{tabular}

The improvement per category and overall improvement are also shown in Figure 6.

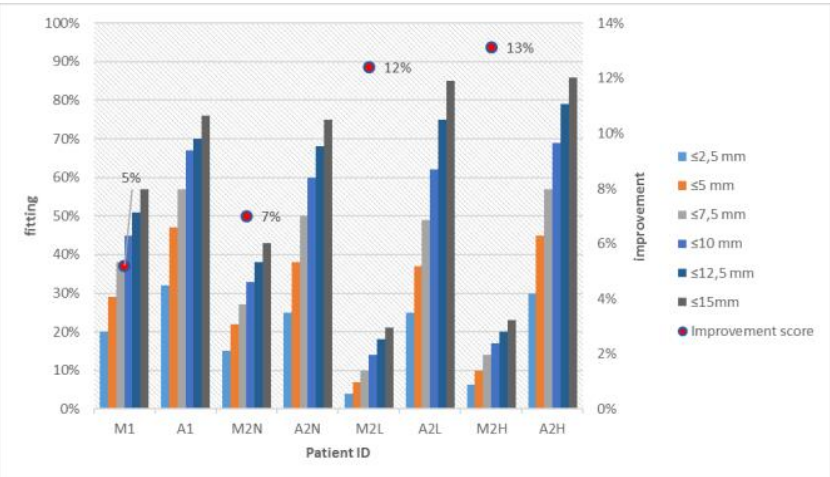

Figure 6 : Summary results, comparing the manual and automatic registration of the model and $3 D$ photo.

\section{Discussion}

The automatic registration of the $3 \mathrm{D}$ photo onto a $\mathrm{CM}$ model delivers improved accuracy in extraction of the electrode positions. All possible solutions, related to the parameters of the initialization phase, are iterated and grant the maximal accuracy of ECG electrode positons.

The automatic registration approach permit a minimal time consumption, a reduction of user intervention and a simple reproduction of the process in different environment and situations .

The automatic electrodes localization is in development phase. A standardization, permitting the generalization of the electrodes detection transparent to the used type, is needed.
In a future version with more control parameters will be incorporated to maximize the fitting to nearby $100 \%$ in range of distance of $<=2.5 \mathrm{~mm}$. Indeed, the incorporation of body tracking while recording, will permit early analyzation of the conditions of the body related to the MC model. It permits filtering $3 \mathrm{D}$ recorded timeframe from the 3D record that are incompatible with the body posture of the $\mathrm{MC}$ model. This will deliver a 3D photo illustrating the closest situation to the MC model.

\section{Conclusion}

The automatic registration of a 3D photo to a patient specific model is better than the manual registration, by permitting a more accurate localization of the ECG electrodes. These accurate electrode location can improve ECG diagnostics and correct lead misplacement errors.

\section{Acknowledgements}

This study was funded by the European Commission through the Eurostars program, grant number 9799 (ALVALE project: Anatomical Localization of the origin of Ventricular Arrhythmias from the 12-Lead ECG).

\section{References}

[1] van Dam PM, Gordon JP, Laks M. Sensitivity of CIPScomputed PVC location to measurement errors in ECG electrode position: the need for the 3D Camera. Journal of Electrocardiology. 2014;47(6):788-93.

[2] van Dam PM, Oostendorp TF, Linnenbank AC, van Oosterom A. Non-invasive imaging of cardiac activation and recovery. Ann Biomed Eng. 2009 Sep;37(9):1739-56.

[3] van Dam PM, Maan AC, van der Putten NHJJ, Bruining N, Dijk WA, Laks M. New Computer Program for detecting 12 Lead ECG Misplacement using a 3D Kinect Camera. In: Murray A, editor. Computing in cardiology; Zaragoza2013. p. $1175-8$.

[4] van Dam PM, Boyle NG, Laks MM, Tung R. Localization of premature ventricular contractions from the papillary muscles using the standard 12-lead electrocardiogram: a feasibility study using a novel cardiac isochrone positioning system. Europace. 2016;18(suppl 4):iv16-iv22.

[5] Lange B, Chang CY, Suma E, Newman B, Rizzo AS, Bolas M. Development and evaluation of low cost game-based balance rehabilitation tool using the Microsoft Kinect sensor. Conf Proc IEEE Eng Med Biol Soc. 2011;2011:1831-4.

Address for correspondence.

Samir Alioui

Weiland 38, 2415BC Nieuwerbrug, The Netherlands

E-mail address: $\underline{\text { samir.alioui@ @eacs.nl }}$ 